\title{
Ipilimumab and Nivolumab as First-Line Treatment of Patients with Renal Cell Carcinoma: The Evidence to Date
}

This article was published in the following Dove Press journal: Cancer Management and Research

\section{Iris Y Sheng \\ Moshe C Ornstein (iD)}

Department of Hematology and Medical Oncology, Cleveland Clinic Taussig Center Institute, Cleveland, OH, USA
Correspondence: Moshe C Ornstein

Tel + I 216-445-6592

Fax + I 216-444-9464

Email ornstem@ccf.org

\begin{abstract}
Immunotherapy has revolutionized the management of metastatic renal cell carcinoma with four checkpoint inhibitors (nivolumab, ipilimumab, avelumab, and pembrolizumab) approved either as monotherapy or as combination therapy. The use of ipilimumab and nivolumab for treatment-naïve, intermediate to poor risk, metastatic renal cell carcinoma was the first checkpoint inhibitor-based combination therapy and remains the only dual checkpoint inhibitor combination approved in mRCC. In this article, we review the trials that led to the approval of ipilimumab and nivolumab in this setting. We also highlight the ongoing trials using this combination, its use in special populations, and clinically relevant unanswered questions.
\end{abstract}

Keywords: ipilimumab, nivolumab, metastatic renal cell carcinoma, immunotherapy, kidney cancer, checkpoint inhibitors

\section{Introduction}

Kidney cancer is one of the top ten most common cancers, with an estimated 73,820 new cases in 2019. ${ }^{1}$ At presentation, $16-25 \%$ of patients will have de novo metastatic disease, ${ }^{2}$ and an estimated $10-28 \%$ of patients with early stage disease will progress to metastatic disease despite local treatment. The difference in outcome between localized disease and metastatic disease is drastic with 5 year survival rates of $92.5 \%$ to $12.0 \%$, respectively. ${ }^{3}$

The most common form of kidney cancer is renal cell carcinoma (RCC) which is known to be highly immunosensitive. The immunogenic nature of metastatic RCC (mRCC) was the rationale for the use of high dose interleukin-2 (IL-2) in these patients, though response rates were poor (objective response rates of $14-25 \%$ with complete response rates of $2-7 \%$ ), and treatment related side effects were fairly toxic. $^{4-7}$

Subsequently, agents targeting the vascular endothelial growth factor (VEGF) and mammalian target of rapamycin (mTOR) pathways, as well as checkpoint inhibitor (CPI) immunotherapy have become the primary treatment option for mRCC patients. Notably, in April 2018 the FDA approved the combination of ipilimumab (CTLA-4 antibody) and nivolumab (PD-1 antibody) for treatment-naïve intermediate and poor risk $\mathrm{mRCC}$. This combination was the first CPI-based combination therapy approved and remains the only dual checkpoint blockade approved in mRCC. In this review, we address the evolution of the combination of ipilimumab and nivolumab in the 
treatment of mRCC. We also highlight ongoing trials with this combination, its use in special populations, and clinically relevant unanswered questions.

\section{Nivolumab and Ipilimumab Monotherapy Trials \\ Nivolumab}

Nivolumab is a monoclonal antibody that selectively blocks the programmed death-1 (PD-1) transmembrane protein on T cells, B cells and NK cells, which activates the immune system and promotes apoptosis. ${ }^{8}$ In the Phase 1 study of nivolumab, CheckMate 003, 34 pretreated patients $(\geq 1$ systemic therapy) with metastatic clear cell renal cell carcinoma (mccRCC) were included. ${ }^{9}$ The overall response rates (ORR) ranged from $24 \%$ in patients receiving $1 \mathrm{mg} / \mathrm{kg}$ to $31 \%$ in those receiving $10 \mathrm{mg} / \mathrm{kg}$. Importantly, 5 patients sustained a response for greater than a year. Fourteen percent of patients experienced grade 3 or higher treatment related adverse events (TRAE); most commonly diarrhea, rash, and pruritis. Only $5 \%$ of patients discontinued therapy because of intolerability. The updated analysis of the phase 1 data demonstrated that at a minimum follow up of 63.9 months, $29 \%$ of patients had a response. ${ }^{10}$ Median duration of response was 12.9 months (95\% CI 8.4-not estimable). Median OS was 22.4 months (95\% CI 12.5-48.6 months) with survival curves plateauing around 3 years.

Given the efficacy and safety established in CheckMate 003, the Phase 2 Checkmate 010 trial investigated nivolumab at $0.3 \mathrm{mg} / \mathrm{kg}, 2 \mathrm{mg} / \mathrm{kg}, 10 \mathrm{mg} / \mathrm{kg}$ in patients with mccRCC, who were previously treated with VEGF directed therapy. ${ }^{11}$ In the 268 patients randomized, nivolumab was active across all cohorts (ORR 20\%, 22\%, and 20\%, respectively) and no dose-response relationship was detected by median progression-free survival (PFS, 2.7 months, 4.0 months, and 4.2 months, respectively, $\mathrm{p}=0.9$ ). McDermott et al presented the 3 year follow up for CheckMate 010, noting the ORR remained at $21 \%$ and the 3 year OS rate was $41 \%$. $^{12}$

Based on these data, the Phase III CheckMate 025 trial randomized patients with mccRCC previously treated with anti-angiogenic therapy to receive nivolumab $3 \mathrm{mg} / \mathrm{kg} \mathrm{IV}$ every 2 weeks versus everolimus $10 \mathrm{mg}$ oral daily (Table 1). ${ }^{13}$ Of the 821 patients who were randomized, patients who received nivolumab had a better ORR $(25 \%$ vs $5 \%, \mathrm{p}<0.001)$ and longer median OS (25 months vs 19.6 months, HR 0.73 ; 95\% CI $0.57-0.93$; $\mathrm{p}=0.002$ ) but no clear difference in PFS $(p=0.11)$. Nivolumab was better tolerated than everolimus with fewer grade 3 or higher

Table I Pivotal Trials That Led to Ipilimumab and Nivolumab Approval

\begin{tabular}{|c|c|c|c|c|c|c|}
\hline Trial & $\mathbf{N}$ & Treatment & $\begin{array}{l}\text { Median Follow- } \\
\text { Up (Months) }\end{array}$ & $\begin{array}{l}\text { Median OS } \\
\text { (Months) }\end{array}$ & $\begin{array}{l}\text { Median PFS } \\
\text { (Months) }\end{array}$ & ORR/CR \\
\hline \multicolumn{7}{|c|}{ Checkpoint inhibitor monotherapy } \\
\hline $\begin{array}{l}\text { CheckMate } \\
025\end{array}$ & 821 & $\begin{array}{l}\text { Nivolumab vs Everolimus } \\
\text { (VEGF-refractory pts) }\end{array}$ & 24 & $\begin{array}{l}25.0 \text { vs } 19.6 \\
(H R 0.73 ; 95 \% \mathrm{Cl} \\
0.57-0.93, p=0.002)\end{array}$ & $\begin{array}{l}4.6 \text { vs } 4.4 \\
(\mathrm{HR} 0.88 ; 95 \% \mathrm{Cl} \\
0.74-\mathrm{I} .03, \mathrm{p}=0 . \mathrm{II})\end{array}$ & $\begin{array}{l}25 \% \text { vs } 5 \%, \\
\text { CR } 1 \%\end{array}$ \\
\hline \multicolumn{7}{|c|}{ Combination of Checkpoint inhibitors } \\
\hline $\begin{array}{l}\text { CheckMate } \\
214\end{array}$ & 1096 & $\begin{array}{l}\text { Nivolumab + Ipilimumab } \\
\text { vs Sunitinib }\end{array}$ & 25.2 & $\begin{array}{l}\text { Intermediate and } \\
\text { poor risk: } \\
\text { NR vs } 26.0 \\
\text { (HR } 0.63 ; 99.8 \% \mathrm{Cl} \\
0.44-0.89, \mathrm{p}<0.00 \mathrm{I} \text { ) } \\
\text { I2 mo OS: } \\
80 \% \text { vs } 72 \% \\
\text { ITT: } \\
\text { NR vs } 32.9 \\
\text { (HR } 0.68 ; 99.8 \% \mathrm{Cl} \\
0.49-0.95, \mathrm{p}<0.00 \mathrm{I} \text { ) } \\
\text { I2-mo OS: } \\
83 \% \text { vs } 77 \%\end{array}$ & $\begin{array}{l}\text { Intermediate and } \\
\text { poor risk: II.6 vs } 8.4 \\
\text { (HR 0.82; } 99.1 \% \mathrm{Cl} \\
0.64-1.05 . \mathrm{p}=0.03 \text { ) } \\
\text { ITT: } \\
\text { I2.4 vs } 12.3 \\
\text { (HR } 0.98 ; 99.1 \% \mathrm{Cl} \\
0.79-1.23, \mathrm{p}=0.85 \text { ) }\end{array}$ & $\begin{array}{l}\text { Intermediate and } \\
\text { poor risk: } 42 \% \text { vs } 27 \% \\
\text { CR } 9 \% \\
\text { ITT: } \\
39 \% \text { vs } 32 \% \\
\text { CR not listed }\end{array}$ \\
\hline
\end{tabular}

Abbreviations: OS, overall survival; PFS, progression-free survival; ORR, objective response rate; CR, complete response; VEGF, vascular endothelial growth factor; HR, hazard ratio; NR, not reached; ITT, intent to treat. 
TRAE (19\% vs 37\%). In a subgroup analysis, OS favored nivolumab both in patients who were PD-L1 positive ( $\geq 1 \%$ expression) with a median OS of 21.8 months vs 18.8 months (HR 0.79; 95\% CI 0.53-1.17) and those who were PD-L1 negative ( $<1 \%$ expression) with a median OS of 27.4 months vs 21.2 months (HR 0.77 ; 95\% CI 0.60 0.97). Final analysis of CheckMate 025 showed that at a minimum follow up of 64 months, patients treated with nivolumab continued to show a better response $(23 \%$ vs $4 \%$ ) as well as improved survival benefit (25.8 months vs 19.7 months, HR 0.73 ; 95\% CI 0.62-0.85). ${ }^{14}$

Recently, two trials explored the efficacy and tolerability of nivolumab in a "real world" population. The Nivolumab Expanded Access Program looked specifically at patients older than 70 years (subgroup 1) and older than 75 years (subgroup 2). Similar response rates (27\% vs $28 \%), 18$ month survival rates $(23.2 \%$ vs $22.8 \%)$ and safety profiles $(27 \%$ vs $40 \%)$ were noted across all ages. ${ }^{15}$ There were no increased rates of discontinuation in older patients compared to younger patients. Similarly, the NIVOREN GETUG AFU trial evaluated the response to nivolumab in tyrosine kinase (TKI) refractory patients. ${ }^{16}$ Their inclusion criteria was broad, including patients with ECOG $>1(15 \%)$ and poor risk disease $(25.5 \%)$ with variable prior treatments $(85 \%$ prior nephrectomy, $22 \% \geq 2$ prior treatments, $21 \%$ prior mTOR therapy). At a median follow up of 20.9 months, the ORR was $21 \%$ with a 12 month OS rate of $69 \%$. Both these studies showed similar efficacy and safety when compared to CheckMate 025 .

Overall, nivolumab has proven sustainable antitumor activity regardless of PD-L1 status and good tolerability across all ages. It has also demonstrated improved efficacy compared to everolimus in refractory mRCC patients. As a result, in November 2015 the FDA approved the use of nivolumab in $\mathrm{mRCC}$ patients who received prior antiangiogenic therapy.

\section{Ipilimumab}

Ipilimumab is a monoclonal antibody that targets the cytotoxic T- lymphocyte- associated protein 4 (CTLA-4) expressed on the surface of $\mathrm{T}$ regulatory cells and activated $\mathrm{T}$ cells; which causes $\mathrm{T}$ effector cell activation and proliferation as well as reducing regulatory $\mathrm{T}$ cell suppression. ${ }^{8}$ Yang et al conducted a study looking at the efficacy of ipilimumab monotherapy in previously treated mccRCC using either low dose (LD; $3 \mathrm{mg} / \mathrm{kg}$ once followed by $1 \mathrm{mg} / \mathrm{kg}$ every 3 weeks) or high dose (HD; $3 \mathrm{mg} / \mathrm{kg}$ every 3 weeks). ${ }^{17}$ The arms were not compared because of uneven ratios of those who had received prior IL-2 therapy in each cohort. Partial response (PR) was noted in 5\% in the LD arm and 13\% in the HD arm, respectively. However, of those who benefited from ipilimumab ( $n=6)$ across both arms, 4 patients were able to sustain a response for greater than a year.

Thirty-three percent of patients had grade 3 or higher TRAE, notably autoimmune-related enteritis, dermatitis, and endocrine deficiencies. There was an association between TRAE and response rates with $30 \%$ of patients who had a grade 3 or higher TRAE achieving a response to treatment compared to $0 \%$ of patients without a TRAE had a response. However, given the concurrent emerging benefit of nivolumab and ipilimumab combination therapy (as noted in the section below), subsequent investigation of ipilimumab monotherapy was not pursued.

\section{Nivolumab and Ipilimumab Combination Trials}

Given the individual clinical efficacy in $\mathrm{mRCC}$ and efficacy in combination in melanoma, ${ }^{18,19}$ the combination of ipilimumab and nivolumab was subsequently investigated. In the Phase I CheckMate 016 trial, patients with mccRCC were randomized to three arms: N3I1 (nivolumab $3 \mathrm{mg} / \mathrm{kg}+$ ipilimumab $1 \mathrm{mg} / \mathrm{kg}$ ), N1I3 (nivolumab $1 \mathrm{mg} / \mathrm{kg}+$ ipilimu$\mathrm{mab} 3 \mathrm{mg} / \mathrm{kg}$ ), and N3I3 (nivolumab $3 \mathrm{mg} / \mathrm{kg}+$ ipilimumab $3 \mathrm{mg} / \mathrm{kg}$ ), followed by nivolumab $3 \mathrm{mg} / \mathrm{kg}$ every 2 weeks until progression or toxicity. ${ }^{20}$ Of note, the analysis matched patients between the N3I1 and N1I3 arms only due to dose limiting toxicity or progression in the N3I3 arm. These patients included treatment-naïve patients, patients who had prior cytokine therapy, and patients who received prior neoadjuvant or adjuvant therapy for localized disease. At a median follow up of 22.3 months, ORR was equivalent in both arms (40\%) and 1 year OS was comparable $(67.3 \%$ vs 69.6\%). However, complete responses (CR) were only seen in the N3I1 arm (11\% vs $0 \%)$. Higher doses of ipilimumab were also associated with higher toxicity (38.3\% vs $61.7 \%$, respectively). The most common grade 3 or higher TRAE in the N3I1 arm were increased liver function tests and diarrhea. In the N1I3 arm, the most common TRAE were liver dysfunction, colitis, lymphopenia, and fatigue. Treatment discontinuation secondary to TRAE in the N3I1 and N1I3 arms were $10.6 \%$ versus $27.7 \%$, respectively. Updated results continued to show a continued benefit in the N3I1 arm compared to the N1I3 arms in both tolerability (grade 3 TRAE 43\% vs 64\%) and durability (105 weeks vs 79.4 
weeks). ${ }^{21}$ Thus, due to better tolerability and complete response rates, the combination of N3I1 was the recommended dose for subsequent clinical trials.

The randomized phase III CheckMate 214 trial explored investigated the combination of nivolumab $3 \mathrm{mg} / \mathrm{kg}$ and ipilimumab $1 \mathrm{mg} / \mathrm{kg}$ every 3 weeks for four doses followed by nivolumab $3 \mathrm{mg} / \mathrm{kg}$ every 2 weeks versus sunitinib in treatment-naïve patients. ${ }^{22}$ (Table 1) Although all International Metastatic RCC Database Consortium (IMDC) risk groups were included, the primary endpoint was in the IMDC intermediate and poor risk groups. At a median follow up of 25.2 months, the patients receiving ipilimumab and nivolumab had improved response rates $(42 \%, 9 \%$ CR vs $27 \%, 1 \% \mathrm{CR}$; $\mathrm{p}<0.001$ ), median PFS (11.6 mo vs 8.4 mo, HR 0.82, $\mathrm{p}=0.03$ ) and OS (NR vs $26.6 \mathrm{mo}$; HR 0.66 ; $95 \%$ CI 0 .$54-0.80 ; \mathrm{p}<0.0001)$ compared to sunitinib. TRAE rates were similar in both arms ( $93 \%$ vs $97 \%$ ), however there were increased rates of discontinuation because of TRAE in the sunitinib arm (12\% vs $22 \%)$. The most common TRAE in the ipilimumab and nivolumab arm were fatigue, pruritis, diarrhea, rash, and nausea. Of the 436 patients treated with ipilimumab and nivolumab that had an immune mediated TRAE, 35\% of patients required high dose steroids ( $\geq 40 \mathrm{mg}$ prednisone daily). Exploratory analyses showed improved ORR $(\mathrm{p}<0.001)$ and median PFS (HR 0.46, 95\% CI 0.31-0.67). Cella et al looked at the health-related quality of life benefit of the combination of ipilimumab with nivolumab versus sunitinib using the Functional Assessment of Cancer Therapy Kidney Symptom Index-19 (FKSI-19), Functional Assessment of Cancer Therapy-General (FACT-G), and EuroQol five dimensional three level (EQ-5D-3L) assessment tools. ${ }^{23}$ There was a reduction in the risk of deterioration across all patient recorded outcome assessment tools (HR0.54; 95\% CI $0.46-0.63$ to HR $0.75,95 \%$ CI $0.63-0.89$ ).

Long term follow up of Checkmate 214, continues to show improved median PFS (HR0.76, 95\% CI 0.63-0.91; $\mathrm{p}<0.01$ ) and median OS (HR 0.66 ; 95\% CI $0.55-0.80$; $\mathrm{p}<0.0001)$ compared to those treated with sunitinib. ${ }^{24}$ When comparing durability of response to ipilimumab and nivolumab versus sunitinib (ORR $42 \%$ vs $26 \%$; $<<0.0001$ ), $68 \%$ vs $52 \%$ of patients continued to sustain a response at 42 months of follow up. Of those who achieved a complete response on ipilimumab with nivolumab, the median treatment free interval was 34.6 months (0.5-49.7 months). Thus, the combination of nivolumab with ipilimumab has become a standard of care for front-line treatment of patients with intermediate and poor risk mccRCC.

\section{Use of Nivolumab and Ipilimumab in Special Populations Brain Metastases}

Although $10 \%$ of patients with mRCC develop brain metastases, the overwhelming majority of clinical trials exclude these patients from participating. ${ }^{25}$ Given the antitumor activity of ipilimumab and nivolumab on brain metastases in both melanoma and NSCLC, two trials have addressed this patient population in $\mathrm{mRCC}$.

In the GETUG-AFU 26 NIVOREN trial, 73 patients with mRCC and asymptomatic brain metastasis, who progressed on VEGFR therapy, were treated with nivolumab monotherapy. ${ }^{26}$ This study included patients with untreated brain metastasis (cohort A) as well as patients who underwent prior brain radiation (cohort B). At a median follow up of 23.6 months, 28 patients in cohort A had an intracranial ORR of $12 \%$ and a median intracranial PFS of 2.7 months (95\% CI 2.3-4.6 months). All patients with an intracranial response also had concurrent extracranial response. Of note, $51 \%$ of patients required corticosteroid use because of symptoms related to brain metastases. Patients in cohort B had a longer median intracranial PFS (4.8 months, 95\% CI 3.0-8.0) compared to cohort A (2.7 months, 95\% CI 2.3-4.6 months). However, they had a shorter 12-month OS $(58.8 \%$, 95\% CI 40.6-73.2\%) compared to cohort A (67\%, 95\% CI 49.6-79.1\%). Because the intracranial response rates were significantly lower than rates seen in extracranial metastases, the authors concluded that single agent nivolumab has limited activity in patients with untreated brain metastasis.

In CheckMate 920, 28 patients with untreated mRCC and asymptomatic brain metastases were treated with the ipilimumab $3 \mathrm{mg} / \mathrm{kg}$ and nivolumab $1 \mathrm{mg} / \mathrm{kg}$ for 4 cycles followed by nivolumab $480 \mathrm{mg}$ every 4 weeks maintenance for up to 2 years. ${ }^{27}$ At a minimum follow up of 6.47 months, the objective response rate was 29\% (all PR) and the median PFS was 9.0 months (2.9-NE). Intracranial response was not reported. OS analysis remains immature. Seven of the 14 patients who experienced grade 3 or higher TRAE had to discontinue therapy. Like in previous studies, the most common grade 3-4 immune mediated adverse events were diarrhea, colitis, diabetic ketoacidosis, hepatitis, hypophysitis, and rash ( $\mathrm{n}=1$ each). 
In general, ipilimumab and nivolumab has shown favorable antitumor activity in this population and similar rates of adverse events as previous studies with this combination. Thus, the combination of ipilimumab and nivolumab can be considered in this specific population and warrants further investigation. Timing of brain-directed therapy (surgery, radiation, etc.) is not yet defined and should be addressed individually with each patient.

\section{Favorable Risk mRCC}

While the primary endpoint in CheckMate 214 focused on IMDC intermediate and poor risk patients, the authors performed exploratory analyses looking at IMDC favorable risk patients who were included in the trial as well. Although the ORR (29\% vs 52\%) and PFS (15.3 months vs 25.1 months; HR 2.18, $\mathrm{p}<0.001$ ) favored sunitinib therapy, the CR rate ( $11 \%$ vs $6 \%$ ) favored the nivolumab plus ipilimumab arm. ${ }^{22}$ More recently, the 42 month follow up of Checkmate 214 continued to show not only a higher CR rate in the ipilimumab nivolumab arm $(13 \%$ vs $6 \%)$ but also more ongoing responses (69\% vs $54 \%) .{ }^{24}$ It remains unclear if there is a survival benefit (HR 1.19; $95 \% \mathrm{CI}$ $0.77-1.85, \mathrm{p}=0.44$ ), although the authors note the PFS curve for the combination arm is stabilizing while the PFS curve for sunitinib continues to decline. Given these findings, ipilimumab and nivolumab can be considered for patients with favorable-risk mRCC.

\section{Non-Clear Cell Histology}

Non-clear cell (ncc) histology makes up approximately $25 \%$ of diagnosed mRCC. ${ }^{28}$ There is limited prospective data to guide treatment of nccRCC, thus treatments for these subtypes are extrapolated from the ccRCC data. In a meta-analysis of nccRCC treated with PD-1/PD-L1 inhibitors, papillary RCC was the only subtype with significant benefit (ORR 29\%, 1 CR) ${ }^{29}$ Two other retrospective series with nivolumab monotherapy showed similar efficacy, in regard to ORR (20\%, all PR) and median PFS (ranging between 3.5-5.4 months).$^{30,31}$ Given the benefit of combination ipilimumab and nivolumab therapy in mccRCC, Gong et al published a retrospective analysis of mnccRCC receiving ipilimumab and nivolumab and noted a best ORR of $33.3 \%$ (no CR) and a median PFS of 7.1 months. ${ }^{28}$ Another series of 18 patients who were treated with ipilimumab and nivolumab for nccRCC at various lines of therapy showed that this combination was feasible with strong anti-tumor activity (33\% PR, $17 \% \mathrm{SD}){ }^{32}$
Currently, there are two phase II trials investigating the role of ipilimumab and/or nivolumab in nccRCC. One trial compares nivolumab plus ipilimumab versus sunitinib in nccRCC (NCT 03075423) 33 $^{33}$ and the other investigates sequential treatment with single agent nivolumab followed by combination therapy in metastatic or unresectable nccRCC. ${ }^{34}$ Results from these trials should help direct CPI therapy in this population.

\section{RCC with Sarcomatoid and Rhabdoid Features}

Sarcomatoid and rhabdoid differentiation can occur in both ccRCC and nccRCC and is associated with a poor prognosis. Tumors with this particular histology are thought to be more immunotherapy responsive because of increased expression of PD-L1 and PD-L1, as well mutations in p53 and BAP1 ${ }^{35-37}$ In a post hoc exploratory analysis of CheckMate 214, 214 patients were identified to have sarcomatoid RCC (sRCC), of which 112 patients had intermediate to poor risk features. ${ }^{38}$ At a minimum follow up of 30 months, there was an improvement in ORR $(56.7 \%$ vs $19.2 \%, \mathrm{p}<0.001)$, median PFS (8.4 mo vs 4.9 mo, HR $0.61,95 \%$ CI $0.38-0.97$, $\mathrm{p}<0.03)$ and median OS (31.2 mo vs $13.6 \mathrm{mo}$, HR 0.55 , $95 \%$ CI $0.33-0.90, \mathrm{p}<0.0155)$ in the ipilimumab with nivolumab arm compared to sunitinib. Notably, while there were no CRs in the sunitinib arm, there was a $18.3 \%$ CR rate in the ipilimumab with nivolumab arm. More recently, a retrospective review comparing outcomes of immunotherapy versus tyrosine kinase inhibitor therapy in patients with RCC and pure sarcomatoid, pure rhabdoid, or mixed sarcomatoid with rhabdoid features, showed that those treated with immunotherapy had better OS (31.4 vs 17.8 months, $\mathrm{p}<0.001) .{ }^{39}$ Given the observed responsiveness of sarcomatoid and/or rhabdoid RCC to immunotherapy, ${ }^{40-44}$ multiple clinical trials are including this rare subtype. ${ }^{45,46}$ (NCT 03866382 , NCT0 3793166).

\section{Immunotherapy Refractory Patients}

While immunotherapy combination therapies are the preferred front-line treatment for ccRCC, there is limited knowledge of the effectiveness and tolerability of immunotherapy as salvage therapy in patients who previously received CPI therapy. In metastatic melanoma refractory to PD-1 therapy, the addition of ipilimumab to nivolumab improved response rates compared to the use of nivolumab alone (16-21\%). ${ }^{47}$ However, there was minimal difference in one year OS rates (54\% vs 55\%). More recently, 
a multi-center series evaluated 30 patients with immunotherapy refractory $\mathrm{mRCC}$ who received ipilimumab and nivolumab as salvage therapy. Most of these patients were IMDC intermediate risk $(60 \%)$ with a median number of 3 prior systemic therapies. At the time of restaging scans, $17 \%$ of patients had a partial response and $3 \%$ had stable disease. ${ }^{48}$ Immune-related TRAE occurred in $37 \%$ of patients with only $6 \%$ of patients having a $\geq$ grade 3 reaction. There are currently multiple trials looking at whether adding an ipilimumab boost to patients refractory to front-line nivolumab is beneficial (NCT03117309, NCT03297593, NCT03203473). ${ }^{49-51}$

\section{Unanswered Questions}

\section{Best Front-Line Therapy}

Currently there are two frontline immunotherapyantiangiogenic combination therapies approved for advanced ccRCC. The society for Immunotherapy of Cancer consensus found that for patients with good performance statue (ECOG 0) and intermediate/poor IMDC risk group stratification, $78 \%$ of panelists would recommend initial treatment with ipilimumab and nivolumab, whereas $17 \%$ of panelists would recommend pembrolizumab with axitinib (Pembro/Axi) ${ }^{52}$ With the analyses available so far, ipilimumab with nivolumab continues has a better CR compared to pembrolizumab with axitinib in the intent to treat population $(11 \%$ vs $6 \%){ }^{24,43}$ However, ipilimumab with nivolumab is also considered a more toxic regimen with $35 \%$ of patients requiring high dose steroids ( $\geq 40 \mathrm{mg}$ / day of prednisone equivalent) for treatment related adverse events and a discontinue rate of $22 \%$ because of intolerable side effects. ${ }^{22}$ Ipilimumab with nivolumab should be considered frontline treatment in those who can tolerate it. A prospective trial comparing the two combinations is needed to clarify this issue; until which either Ipi/Nivo or Pembro/Axi combination therapy is reasonable.

\section{Duration of Therapy}

It remains unclear how long patients should remain on therapy in order to obtain and sustain optimal clinical benefit. In general, patients continue on treatment until toxicity or progression. Clinical trials of CPI in melanoma have showed durable responses in patients who discontinued therapy for reasons other than disease progression. Updates from Keynote 006 showed that $78.4 \%$ of patients who completed 2 years of pembrolizumab therapy continued to have disease control at 5 years. ${ }^{53}$ The estimated risk for progression or death nearly 10 months after completing pembrolizumab was $9 \%$ and did not differ by best response to pembrolizumab. Similarly, the long term update of the phase III Checkmate 067 trial, showed that $71 \%$ of patients with advanced melanoma who received ipilimumab with nivolumab did not need subsequent therapy at 4 years. ${ }^{18}$

Similar trends have been seen in RCC as well. In the extended follow up analysis of CheckMate $214,52 \%$ of patients with intermediate to poor risk disease who were treated with nivolumab/ipilimumab had a response duration $\geq 18$ months with a median time to response of 2.8 months (range 2.7-3.1 months). ${ }^{54}$ Of those who responded but discontinued therapy for reasons other than progression, more patients who were treated with the combination therapy were able to remain off of therapy compared to those treated with sunitinib (38\% vs $26 \%$ ). Further studies into the biology of the tumor and biomarkers of response are needed to help predict who will not only benefit from therapy but also sustain a response once therapy is discontinued.

Likewise, studies looking into intermittent immunotherapy dosing have begun. In a small prospective phase II trial of intermittent nivolumab monotherapy in 14 VEGFR TKI refractory aRCC, demonstrated that $80 \%$ of eligible patients were able to sustain a response at 48 weeks post treatment suspension. ${ }^{55}$ Currently, there are two phase trials investigating this question. (Table 2) One trial includes patients with treatment-naïve mRCC who will be treated with ipilimumab and nivolumab followed by 24 weeks of nivolumab maintenance, at which point patients with a CR or PR will enter an observation phase until progression (NCT03126331). ${ }^{56}$ In another trial, treatment-naïve mRCC patients with advanced RCC will receive nivolumab monotherapy with a transition to either therapy suspension ( $\operatorname{arm} A$ ) for those with a persistent CR/ PR versus a N3I1 boost (arm B) for those with PD. (NCT03203473). ${ }^{50}$ Novel dosing schedules, early discontinuation considerations, and biomarkers of response are all sorely needed to identify patients who can sustain disease regression while off of therapy.

\section{Restarting Therapy in Patients Who Developed Immune-Related Adverse Events}

A host of data suggests that the development of immunerelated adverse events (irAEs) are associated with improved response to CPI therapy. A meta-analysis of 48 clinical trials using ipilimumab and nivolumab in various solid tumors found that the ORR of combination therapy 
Table 2 Ongoing Trials Using Ipilimumab and Nivolumab in Clear Cell Advanced RCC

\begin{tabular}{|c|c|c|c|c|c|}
\hline NCT \# & Phase & $\mathbf{N}$ & Treatment & Primary Endpoint & Status \\
\hline \multicolumn{6}{|c|}{ Sequencing Therapy in ccRCC } \\
\hline $03117309^{51}$ & II & 120 & $\begin{array}{l}\text { Nivolumab } 240 \mathrm{mg} \text { IV every } 2 \text { weeks } \times 6 \text { doses then } \\
360 \mathrm{mg} \text { IV every } 3 \text { weeks for up to } 84 \text { weeks. } \\
\text { For PD anytime or SD at I2 months, Nivolumab } \\
\text { will be increased to } 3 \mathrm{mg} / \mathrm{kg} \text { every } 3 \text { weeks } \times 4 \\
\text { doses with Ipilimumab I mg/kg every } 3 \text { weeks } \times 4 \\
\text { doses. When Ipilimumab is completed, Nivolumab } \\
\text { will be reverted back to } 360 \mathrm{mg} \text { IV every } 3 \text { weeks } \\
\text { for up to } 48 \text { weeks. }\end{array}$ & PFS & Recruiting \\
\hline $03297593^{49}$ & II & 74 & $\begin{array}{l}\text { Nivolumab } 240 \mathrm{mg} \text { every } 2 \text { weeks for first } 20 \text { weeks } \\
\text { then continued on } 480 \mathrm{mg} \text { every } 4 \text { weeks after. } \\
\text { After } 2 \text { weeks of Nivolumab, Ipilimumab I mg/kg } \\
\text { every } 6 \text { weeks will be added until PR or CR is } \\
\text { reached. Once PR/CR is reached, Ipilimumab will be } \\
\text { discontinued and Nivolumab monotherapy will } \\
\text { continue. }\end{array}$ & ORR & Recruiting \\
\hline $03126331^{56}$ & II & 40 & $\begin{array}{l}\text { Frontline Ipilimumab and Nivolumab for } 4 \text { doses } \\
\text { then } 24 \text { weeks of maintenance. Patients with a CR/ } \\
\text { PR will enter an observation. }\end{array}$ & $\begin{array}{l}\text { Proportion of patients who receive } \\
\text { intermittent therapy and rate of participants } \\
\text { who maintain off therapy for at least } 9 \\
\text { months }\end{array}$ & Recruiting \\
\hline $03203473^{50}$ & II & 58 & $\begin{array}{l}\text { Upfront Nivolumab monotherapy with a transition } \\
\text { to either therapy suspension }(\operatorname{arm} A) \text { for those with } \\
\text { a persistent CR/PR versus an Ipilimumab }+ \\
\text { Nivolumab boost (arm B) for those with PD }\end{array}$ & $\begin{array}{l}\text { Number of subjects with persistent PR or CR } \\
\text { at I year and number of subjects with SD/PD } \\
\text { that convert to PR/CR with boost }\end{array}$ & $\begin{array}{l}\text { Active, } \\
\text { not } \\
\text { recruiting }\end{array}$ \\
\hline \multicolumn{6}{|c|}{ Combinations with other therapies } \\
\hline $03793166^{45}$ & III & 1046 & $\begin{array}{l}\text { Ipilimumab + Nivolumab for } 4 \text { cycles followed by } \\
\text { Nivolumab monotherapy (arm A) or combination of } \\
\text { Nivolumab Cabozantinib (arm B) for non-CR/non- } \\
\text { PD }\end{array}$ & OS & Recruiting \\
\hline $03065179^{66}$ & II & 25 & $\begin{array}{l}\text { Ipilimumab+ Nivolumab + SBRT to I-2 metastatic } \\
\text { sites }\end{array}$ & Safety & Recruiting \\
\hline $03552380^{67}$ & II & 53 & $\begin{array}{l}\text { Entinostat: } 5 \mathrm{mg}, 3 \mathrm{mg} \text {, or } 2 \mathrm{mg} \text { orally on DI, } 8,15+ \\
\text { Nivolumab+ Ipilimumab }\end{array}$ & Dose finding and ORR & Recruiting \\
\hline $03937219^{68}$ & III & 676 & $\begin{array}{l}\text { Cabozantinib + Nivolumab + Ipilimumab ( } 4 \text { doses) } \\
\text { followed by Cabozantinib + Nivolumab vs } \\
\text { Cabozantinib-matched placebo + Nivolumab }+ \\
\text { Ipilimumab ( } 4 \text { doses) followed by Cabozantinib- } \\
\text { matched placebo + Nivolumab }\end{array}$ & PFS & Recruiting \\
\hline
\end{tabular}

Abbreviations: PFS, progression-free survival; ORR, objective response rate; OS, overall survival; D, day; IV, intravenous; CR, complete response; PR, partial response; SBRT, stereotactic body radiation therapy.

was positively correlated with irAE of the skin ( $\mathrm{r}=0.54$, $\mathrm{p}=0.04)$ and GI tract $(\mathrm{r}=0.60, \mathrm{p}=0.02) .{ }^{57}$ Specifically, in RCC, the NIVOREN GETUG AFU trial found that those who had a grade $\geq 3$ TRAE (18\%) had a longer PFS (HR $0.69,95 \%$ CI $0.56-0.86$ ) than those who did not. ${ }^{16}$
IrAEs usually occur within 3-6 months of CTLA-4 or PD-L1 initiation, however they can occur at any time during treatment. ${ }^{58}$ Severity is determined by the common terminology criteria for adverse events grading system. ${ }^{59}$ Across $\mathrm{ASCO}^{60}$ and NCCN guidelines, ${ }^{61}$ grade $2-3$ irAEs 
can be managed with temporary discontinuation of therapy until symptoms resolve below grade 1 or patients are on a maximum of prednisone $10 \mathrm{mg}$ daily. Grade 4 irAEs should lead to permanent discontinuation of combination therapy.

In CheckMate 214, $47 \%$ of patients experienced a TRAE on ipilimumab and nivolumab therapy, with diarrhea $(4 \%)$ and hepatitis (4\%) being the most common. ${ }^{22}$ Twenty two percent of these patients discontinued therapy secondary to AE intolerability. While patients who experienced a severe irAE on protocol were not allowed to continue with nivolumab monotherapy, the NCCN guidelines suggest that patient can be restarted on PD-1 or PDL1 monotherapy after symptoms secondary to combination therapy resolve. ${ }^{61}$ Currently, the Phase $3 \mathrm{~b} / 4$ CheckMate 920 trial is looking to answer this question by evaluating the incidence of high grade irAEs with patients treated with both ipilimumab and nivolumab (NCT02982954). ${ }^{62}$ Additional data is needed to guide therapy resumption in patients who develop severe irAEs.

\section{Treating Patients with Underlying Autoimmune Conditions}

Patients with active autoimmune conditions have been excluded from the ipilimumab and nivolumab trials, given the theoretical exacerbation of their underlying disorder when the CTLA-4 and PD-1 receptors are blocked. In a retrospective study of PD-1 blockers (pembrolizumab or nivolumab) for advanced melanoma, 52 patients with underlying autoimmune conditions had 33\% response rates and $38 \%$ had a flare of their pre-existing disease requiring immunosuppression. ${ }^{63}$ Of note, only 2 patients had to discontinue treatment due to their flare. However, $29 \%$ developed other iRAEs, with $8 \%$ of these patients discontinuing treatment. Similarly, in a retrospective study of 30 patients with advanced melanoma and a preexisting autoimmune condition ( $43 \%$ of which were on immunosuppressive therapy) were treated with ipilimumab, there were $20 \%$ response rates with $27 \%$ exacerbation of their underlying condition requiring steroid management. ${ }^{64}$ There have been no large series published in renal cell carcinoma or with the combination of ipilimumab with nivolumab. However, the Society of Immunotherapy of Cancer consensus recommends that patients with aRCC can be considered for ipilimumab nivolumab combination therapy if they do not have a life threatening autoimmune condition or require immunosuppressive treatments. ${ }^{52}$

\section{Identifying Patients for Nivolumab Monotherapy in} Treatment-Naïve mRCC

While the combination of ipilimumab and nivolumab has proven survival benefits, the tolerability of the regimen has been a concern in clinical practice. A metanalysis using the World Health Organization pharmacovigilance database showed that combination therapy (CTLA- 4 plus anti PD-1 therapy) is associated with more iRAE compared to anti-PD-1 monotherapy (55-60\% vs $10-20 \%$ ) across multiple tumor types. ${ }^{65}$ Thus, investigations into nivolumab monotherapy upfront is warranted.

In the phase II TITAN-RCC trial, treatment-naïve (cohort 1) and VEGF-refractory (cohort 2) patients were induced with nivolumab $240 \mathrm{mg}$ IV every 2 weeks for 4 doses, at which point they either received a N3I1 boost for early progression or continued on nivolumab $240 \mathrm{mg}$ IV every 2 week maintenance. This reassessment was repeated again after the 8th dose. Specifically, in cohort 1 , the ORR was worse in the nivolumab monotherapy arm compared to those who received an N3I1 boost (29\% vs 37\%). Of those who had a N3I1 boost, $29.8 \%$ of patients had improvement after the combination was given. Of those who responded with a PR (27\%) or CR (2\%) at 4 weeks, median PFS was not reached at analysis. OS data remains immature. This study highlights that there is a small subset of patients that may not need combination therapy upfront and can respond when ipilimumab is added later. There are currently multiple trials investigating this question (Table 2). ${ }^{51}$

\section{Conclusion}

The combination of ipilimumab and nivolumab is approved in front-line intermediate and poor risk mRCC on the basis of improved OS compared to sunitinib. The benefits of this combination is also noted in subpopulations such as those who have brain metastases, favorable risk profile, and non-clear cell histology. Although a multitude of questions remain unanswered, ongoing clinical trials will serve to guide the use of ipilimumab and nivolumab and refine its application across mRCC patients.

\section{Disclosure}

Moshe C. Ornstein reports grant support from Pfizer, BMS; speaking: BMS, Exelixis; education support: Pfizer; consulting: BMS, Pfizer, Exelixis. The authors report no other conflicts of interest in this work. 


\section{References}

1. Key Statistics About Kidney Cancer. Available from: https://www. cancer.org/cancer/kidney-cancer/about/key-statistics.html. Accessed December 24, 2019.

2. Kidney Cancer - Statistics. Cancer.Net. [updated June 25, 2012]. Available from: https://www.cancer.net/cancer-types/kidney-cancer /statistics. Accessed December 24, 2019.

3. Cancer of the kidney and renal pelvis - cancer stat facts. SEER. Available from: https://seer.cancer.gov/statfacts/html/kidrp.html Accessed December 24, 2019.

4. Fyfe G, Fisher RI, Rosenberg SA, Sznol M, Parkinson DR, Louie AC. Results of treatment of 255 patients with metastatic renal cell carcinoma who received high-dose recombinant interleukin-2 therapy. $J$ Clin Oncol. 1995;13(3):688-696. doi:10.1200/JCO.1995.13.3.688

5. Fisher RI, Rosenberg SA, Fyfe G. Long-term survival update for high-dose recombinant interleukin-2 in patients with renal cell carcinoma. Cancer J Sci Am. 2000;6(Suppl 1):S55-57.

6. Yang JC, Sherry RM, Steinberg SM, et al. Randomized study of high - dose and low - dose inter leukin - 2 in patients with metastatic renal cancer. $J$ Clin Oncol. 2003;21(16):3127-3132. doi:10.1200/ JCO.2003.02.122

7. McDermott DF, Cheng S-C, Signoretti S, et al. The high-dose aldesleukin "select" trial: a trial to prospectively validate predictive models of response to treatment in patients with metastatic renal cell carcinoma. Clin Cancer Res. 2015;21(3):561-568. doi:10.1158/ 1078-0432.CCR-14-1520

8. Buchbinder EI, Desai A. CTLA-4 and PD-1 pathways: similarities, differences, and implications of their inhibition. Am J Clin Oncol. 2016;39(1):98-106. doi:10.1097/COC.0000000000000239

9. Topalian SL, Hodi FS, Brahmer JR, et al. Safety, activity, and immune correlates of anti-PD-1 antibody in cancer. $N$ Engl $J$ Med. 2012;366(26):2443-2454. doi:10.1056/NEJMoa1200690

10. Topalian SL, Hodi FS, Brahmer JR, et al. Five-year survival and correlates among patients with advanced melanoma, renal cell carcinoma, or non-small cell lung cancer treated with nivolumab. JAMA Oncol. 2019; e192187. doi:10.1001/jamaoncol.2019.2187.

11. Motzer RJ, Rini BI, McDermott DF, et al. Nivolumab for metastatic renal cell carcinoma: results of a randomized phase II trial. $J$ Clin Oncol. 2015;33(13):1430-1437. doi:10.1200/JCO.2014.59.0703

12. McDermott DF, Motzer RJ, Atkins MB, et al. Long-term overall survival (OS) with nivolumab in previously treated patients with advanced renal cell carcinoma (aRCC) from phase I and II studies. JCO. 2016;34 (15_suppl):4507. doi:10.1200/JCO.2016.34.15_suppl.4507

13. Motzer RJ, Escudier B, McDermott DF, et al. Nivolumab versus everolimus in advanced renal-cell carcinoma. $N$ Engl $J$ Med. 2015;373(19):1803-1813. doi:10.1056/NEJMoa1510665

14. Motzer RJ, Tykodi SS, Escudier B, et al. Final analysis of the CheckMate 025 trial comparing nivolumab (NIVO) versus everolimus (EVE) with $>5$ years of follow-up in patients with advanced renal cell carcinoma (aRCC). JCO. 2020;38(6_suppl):617. doi:10.1200/JCO.2020.38.6_suppl.617

15. Vitale MG, Scagliarini S, Galli L, et al. Efficacy and safety data in elderly patients with metastatic renal cell carcinoma included in the nivolumab expanded access program (EAP) in Italy. PLoS One. 2018;13(7):e0199642. doi:10.1371/journal.pone.0199642

16. Laurence Albiges SN, Ci le Dalban CC, Medical Oncology GR, et al. Safety and efficacy of nivolumab in metastatic renal cell carcinoma (mRCC): final analysis from the NIVOREN GETUG AFU 26 study. ASCO Meeting library. February 2019. Available from: https://meet inglibrary.asco.org/record/170207/abstract. Accessed February 28, 2019.

17. Yang JC, Hughes M, Kammula U, et al. Ipilimumab (Anti-CTLA4 antibody) causes regression of metastatic renal cell cancer associated with enteritis and hypophysitis. J Immunother. 2007;30(8):825-830. doi:10.1097/CJI.0b013e318156e47e
18. Larkin J, Chiarion-Sileni V, Gonzalez R, et al. Five-year survival with combined nivolumab and ipilimumab in advanced melanoma. $N$ Engl J Med. 2019;381(16):1535-1546. doi:10.1056/NEJMoa 1910836

19. Wolchok JD, Kluger H, Callahan MK, et al. Nivolumab plus ipilimumab in advanced melanoma. N Engl J Med. 2013;369(2):122-133. doi:10.1056/NEJMoa1302369

20. Hammers HJ, Plimack ER, Infante JR, et al. Safety and efficacy of nivolumab in combination with ipilimumab in metastatic renal cell carcinoma: the CheckMate 016 study. JCO. 2017;35(34):3851-3858. doi:10.1200/JCO.2016.72.1985

21. Hammers H, Plimack ER, Infante JR, et al. Updated results from a phase I study of nivolumab (Nivo) in combination with ipilimumab (Ipi) in metastatic renal cell carcinoma (mRCC): the CheckMate 016 study. Ann Oncol.2016;27(suppl_6):vi364. doi:10.1093/annonc/mdw378.16

22. Motzer RJ, Tannir NM, McDermott DF, et al. Nivolumab plus ipilimumab versus sunitinib in advanced renal-cell carcinoma. $N$ Engl J Med. 2018;378(14):1277-1290. doi:10.1056/NEJMoa1712126

23. Cella D, Grünwald V, Escudier B, et al. Patient-reported outcomes of patients with advanced renal cell carcinoma treated with nivolumab plus ipilimumab versus sunitinib (CheckMate 214): a randomised, phase 3 trial. Lancet Oncol. 2019;20(2):297-310. doi:10.1016/S14702045(18)30778-2

24. Tannir NM, McDermott DF, Escudier B, et al. Overall survival and independent review of response in CheckMate 214 with 42-month follow-up: first-line nivolumab + ipilimumab $(\mathrm{N}+\mathrm{I})$ versus sunitinib (S) in patients (pts) with advanced renal cell carcinoma (aRCC). JCO. 2020;38(6_suppl):609. doi:10.1200/JCO.2020.38.6_suppl.609

25. Cagney DN, Martin AM, Catalano PJ, et al. Incidence and prognosis of patients with brain metastases at diagnosis of systemic malignancy: a population-based study. Neuro-Oncology. 2017;19 (11):1511-1521. doi:10.1093/neuonc/nox077

26. Flippot R, Dalban C, Laguerre B, et al. Safety and efficacy of nivolumab in brain metastases from renal cell carcinoma: results of the GETUG-AFU 26 NIVOREN multicenter phase II study. $J$ Clin Oncol. 2019;37(23):2008-2016. doi:10.1200/JCO.18.02218

27. Emamekhoo H, Olsen M, Carthon BC, et al. Safety and efficacy of nivolumab plus ipilimumab (NIVO+IPI) in patients with advanced renal cell carcinoma (aRCC) with brain metastases: interim analysis of CheckMate 920. JCO. 2019;37(15_suppl):4517. doi:10.1200/ JCO.2019.37.15_suppl.4517

28. Dizman N Clinical activity of ipilimumab plus nivolumab in patients with metastatic non-clear cell RCC. Practice update. [updated December 23, 2019]. Available from: https://www.practiceupdate.com/content/clinicalactivity-of-ipilimumab-plus-nivolumab-in-patients-with-metastatic-nonclear-cell-rcc/93720. Accessed December 26, 2019.

29. McKay RR, Bossé D, Xie W, et al. The clinical activity of PD-1/PDL1 inhibitors in metastatic non-clear cell renal cell carcinoma. Cancer Immunol Res. 2018;6(7):758-765. doi:10.1158/2326-6066. CIR-17-0475

30. Koshkin VS, Barata PC, Zhang T, et al. Clinical activity of nivolumab in patients with non-clear cell renal cell carcinoma. J Immunother Cancer. 2018;6(1). doi:10.1186/s40425-018-0319-9

31. Chahoud J, Campbell MT, Gao J, et al. Nivolumab (nivo) for patients (pts) with metastatic non-clear cell renal cell carcinoma (nccRCC): a single-institution experience. JCO. 2018;36(15_suppl):4585. doi:10.1200/JCO.2018.36.15_suppl.4585

32. Gupta R, Ornstein MC, Li H, et al. Clinical activity of ipilimumab plus nivolumab in patients with metastatic non-clear cell renal cell carcinoma - ScienceDirect. Clin Genitourin Cancer. 2019. doi:10.1016/j.clgc.2019.11.012

33. Randomized phase-II study of nivolumab plus ipilimumab vs. standard of care in untreated and advanced non-clear cell RCC - full text view - ClinicalTrials.gov. Available from: https://clinicaltrials.gov/ ct2/show/NCT03075423. Accessed January 19, 2020. 
34. Phase II sequential treatment trial of single agent nivolumab, then combination ipilimumab + nivolumab in metastatic or unresectable non-clear cell renal cell carcinoma (ANZUP1602) - full text view ClinicalTrials.gov. Available from: https://clinicaltrials.gov/ct2/show/ NCT03177239. Accessed January 20, 2020.

35. Pichler M, Hutterer G, Chromecki T, et al. External validation of the leibovich prognosis score for nonmetastatic clear cell renal cell carcinoma at a single European center applying routine PubMed NCBI. J Urol. 2011;186(5):1773-1777. doi:10.1016/j.juro.2011.07.034

36. Bakouny Z, Vokes N, Gao X, et al. Efficacy of immune checkpoint inhibitors (ICI) and genomic characterization of sarcomatoid and/or rhabdoid (S/R) metastatic renal cell carcinoma (mRCC). JCO. 2019;37(15_suppl):4514. doi:10.1200/JCO.2019.37.15_suppl.4514

37. Leroy X, Zini L, Buob D, Ballereau C, Villers A, Aubert S. Renal cell carcinoma with rhabdoid features: an aggressive neoplasm with overexpression of p53. Arch Pathol Lab Med. 2007;131(1):102-106. doi:10.1043/1543-2165(2007)131[102:RCCWRF]2.0.CO;2

38. McDermott DF, Choueiri TK, Motzer RJ, et al. CheckMate 214 post-hoc analyses of nivolumab plus ipilimumab or sunitinib in IMDC intermediate/poor-risk patients with previously untreated advanced renal cell carcinoma with sarcomatoid features. JCO. 2019;37(15_suppl):4513. doi:10.1200/JCO.2019.37.15_suppl.4513

39. Samiei A, Tayshetye P, Sanguino A, et al. The clinical outcome of renal cell carcinoma with rhabdoid and sarcomatoid differentiation. JCO. 2019;37(15_suppl):e16083. doi:10.1200/JCO.2019.37.15_suppl.e16083

40. Motzer RJ, Powles T, Atkins MB, et al. IMmotion151: a randomized phase III study of atezolizumab plus bevacizumab vs sunitinib in untreated metastatic renal cell carcinoma (mRCC): journal of clinical oncology: vol 36, no 6_suppl. J Clin Oncol. 2018;36(6):578. doi:10.1200/JCO.2018.36.6_suppl.578

41. Rini BI, Plimack ER, Stus V, et al. Pembrolizumab (pembro) plus axitinib (axi) versus sunitinib as first-line therapy for metastatic renal cell carcinoma ( $\mathrm{mRCC})$ : outcomes in the combined IMDC intermediate/poor risk and sarcomatoid subgroups of the phase 3 KEYNOTE-426 study. JCO. 2019;37(15_suppl):4500. doi:10.1200/ JCO.2019.37.15_suppl.4500

42. Rini BI, Motzer RJ, Powles T, et al. Atezolizumab (atezo) + bevacizumab (bev) versus sunitinib (sun) in pts with untreated metastatic renal cell carcinoma (mRCC) and sarcomatoid (sarc) histology: iMmotion151 subgroup analysis. JCO. 2019;37(15_suppl):4512. doi:10.1200/JCO.2019.37.15_suppl.4512

43. Rini BI, Plimack ER, Stus V, et al. Pembrolizumab plus axitinib versus sunitinib for advanced renal-cell carcinoma. $N$ Engl J Med. 2019;380(12):1116-1127. doi:10.1056/NEJMoa1816714

44. Wynja E, Solomon B, Bleeker J. Complete and prolonged response of renal cell carcinoma with rhabdoid features to checkpoint inhibitor therapy. PubMed NCBI. J Immunother. 2018;41(7):340-342. doi:10.1097/CJI.0000000000000238

45. Immunotherapy with nivolumab and ipilimumab followed by nivolumab or nivolumab with cabozantinib for patients with advanced kidney cancer, the PDIGREE study - full text view - ClinicalTrials. gov. Available from: https://clinicaltrials.gov/ct2/show/ NCT03793166. Accessed January 20, 2020.

46. Testing the effectiveness of two immunotherapy drugs (nivolumab and ipilimumab) with one anti-cancer targeted drug (cabozantinib) for rare genitourinary tumors - full text view - ClinicalTrials.gov. Available from: https://clinicaltrials.gov/ct2/show/NCT03866382. Accessed April 20, 2020.

47. Zimmer L, Apuri S, Eroglu Z, et al. Ipilimumab alone or in combination with nivolumab after progression on anti-PD-1 therapy in advanced melanoma. Eur J Cancer. 2017;75:47-55. doi:10.1016/j. ejca.2017.01.009

48. Gul A, Shah NJ, Mantia C, et al. Ipilimumab plus nivolumab (Ipi/ Nivo) as salvage therapy in patients with immunotherapy (IO)-refractory metastatic renal cell carcinoma (mRCC). JCO. 2019;37(7_suppl):669. doi:10.1200/JCO.2019.37.7_suppl.669
49. Nivolumab in combination with ipilimumab in patients with metastatic renal cell carcinoma - full text view - ClinicalTrials.gov. Available from: https://clinicaltrials.gov/ct2/show/NCT03297593. Accessed January 20, 2020.

50. Study of optimized management of nivolumab based on response in patients with advanced RCC (OMNIVORE study) - full text view ClinicalTrials.gov. Available from: https://clinicaltrials.gov/ct2/show/ NCT03203473. Accessed January 19, 2020.

51. Study of front line therapy with nivolumab and salvage nivolumab + ipilimumab in patients with advanced renal cell carcinoma - full text view - ClinicalTrials.gov. Available from: https:/clinicaltrials.gov/ ct2/show/NCT03117309. Accessed January 19, 2020.

52. Rini BI, McDermott DF, Hammers H, et al. Society for immunotherapy of cancer consensus statement on immunotherapy for the treatment of renal cell carcinoma. J Immunother Cancer. 2016;4(1):81. doi:10.1186/s40425-016-0180-7

53. Robert C, Ribas A, Schachter J, et al. Pembrolizumab versus ipilimumab in advanced melanoma (KEYNOTE-006): post-hoc 5-year results from an open-label, multicentre, randomised, controlled, phase 3 study. Lancet Oncol. 2019;20(9):1239-1251. doi:10.1016/ S1470-2045(19)30388-2

54. Motzer RJ, Rini BI, McDermott DF, et al. Nivolumab plus ipilimumab versus sunitinib in first-line treatment for advanced renal cell carcinoma: extended follow-up of efficacy and safety results from a randomised, controlled, phase 3 trial. Lancet Oncol. 2019;20 (10):1370-1385. doi:10.1016/S1470-2045(19)30413-9

55. Ornstein MC, Wood LS, Hobbs BP, et al. A phase II trial of intermittent nivolumab in patients with metastatic renal cell carcinoma (mRCC) who have received prior anti-angiogenic therapy. J Immunother Cancer. 2019;7(1):127. doi:10.1186/s40425-019-0615-z

56. Intermittent therapy in metastatic renal cell carcinoma patients treated with ipilimumab and nivolumab - full text view - ClinicalTrials.gov. Available from: https://clinicaltrials.gov/ct2/show/NCT03126331. Accessed January 20, 2020.

57. Xing P, Zhang F, Wang G, et al. Incidence rates of immune-related adverse events and their correlation with response in advanced solid tumours treated with NIVO or NIVO+IPI: a systematic review and meta-analysis. J ImmunoTher Cancer. 2019;7(1):341. doi:10.1186/ s40425-019-0779-6

58. Trinh S, Le A, Gowani S, La-Beck NM. Management of immune-related adverse events associated with immune checkpoint inhibitor therapy: a minireview of current clinical guidelines. Asia Pac J Oncol Nurs. 2019;6(2):154-160. doi:10.4103/apjon. apjon_3_19

59. Common terminology criteria for adverse events (CTCAE) $\mid$ protocol development | CTEP. Available from: https://ctep.cancer.gov/ protocolDevelopment/electronic_applications/ctc.htm\#ctc_50. Accessed July 30, 2019.

60. Brahmer JR, Lacchetti C, Schneider BJ, et al. Management of immune-related adverse events in patients treated with immune checkpoint inhibitor therapy: american society of clinical oncology clinical practice guideline. JCO. 2018;36(17):1714-1768. doi:10.1200/JCO.2017.77.6385

61. NCCN. Management of immunotherapy- related toxicity. Available from: https://www.nccn.org/professionals/physician_gls/pdf/immu notherapy.pdf. Accessed January 19, 2020.

62. A study to evaluate the safety of nivolumab and ipilimumab in subjects with previously untreated advanced or metastatic renal cell cancer - full text view - ClinicalTrials.gov. Available from: https:// clinicaltrials.gov/ct2/show/NCT02982954. Accessed January 20, 2020.

63. Menzies AM, Johnson DB, Ramanujam S, et al. Anti-PD-1 therapy in patients with advanced melanoma and preexisting autoimmune disorders or major toxicity with ipilimumab. Ann Oncol. 2017;28 (2):368-376. doi:10.1093/annonc/mdw443 
64. Johnson DB, Sullivan RJ, Ott PA, et al. Ipilimumab therapy in patients with advanced melanoma and preexisting autoimmune disorders. JAMA Oncol. 2016;2(2):234-240. doi:10.1001/jamaoncol.2015.4368

65. Wang DY, Salem J-E, Cohen JV, et al. Fatal toxic effects associated with immune checkpoint inhibitors: a systematic review and meta-analysis. JAMA Oncol. 2018;4(12):1721-1728. doi:10.1001/ jamaoncol.2018.3923

66. Trial of SBRT in combination with nivolumab/ipilimumab in RCC/ kidney cancer patients - full text view - ClinicalTrials.gov. Available from: https://clinicaltrials.gov/ct2/show/NCT03065179. Accessed January 20, 2020.
67. Study of entinostat with nivolumab plus ipilimumab in previously treated renal cell carcinoma - full text view - ClinicalTrials.gov. Available from: https://clinicaltrials.gov/ct2/show/NCT03552380. Accessed January 20, 2020.

68. Study of cabozantinib in combination with nivolumab and ipilimumab in patients with previously untreated advanced or metastatic renal cell carcinoma - full text view - ClinicalTrials.gov. Available from: https://clinicaltrials.gov/ct2/show/NCT03937219. Accessed January 20, 2020.

\section{Publish your work in this journal}

Cancer Management and Research is an international, peer-reviewed open access journal focusing on cancer research and the optimal use of preventative and integrated treatment interventions to achieve improved outcomes, enhanced survival and quality of life for the cancer patient.
The manuscript management system is completely online and includes a very quick and fair peer-review system, which is all easy to use. Visit http://www.dovepress.com/testimonials.php to read real quotes from published authors. 